\title{
Current concepts on the pathophysiology and management of recurrent ankle sprains and chronic ankle instability
}

\author{
C. Collin Herb $\cdot$ Jay Hertel
}

Published online: 9 January 2014

(C) Springer Science + Business Media New York 2014

\begin{abstract}
Lateral ankle sprains occur more than any other musculoskeletal injury in an athletic population. The greatest risk for an inversion ankle sprain is a history of a previous sprain. Recurring instability associated with subjective complaints of giving way is often termed chronic ankle instability (CAI). CAI has been associated with altered postural control, sensorimotor deficits, reduced physical activity, functional limitations, and long-term degeneration of the joint. Alterations, including muscle activity and changes in the kinematics of the lower extremity may predispose this population to continued instability at the joint and long term health issues. Common treatments include using external support, therapeutic modalities, and directed rehabilitation. Wearing ankle braces and performing balance training has been shown to reduce the risk of recurrent ankle sprains. Directed rehabilitation may improve function in patients with CAI. By identifying and minimizing dysfunction following ankle sprains, clinicians may improve the short-term and longterm function of this population.
\end{abstract}

Keywords Rehabilitation - Sports medicine $\cdot$ Motor control

\section{Introduction}

In the United States there are an estimated 1,600,000 physician visits and 8,000 hospitalizations per year due to lateral ankle sprains [1]. The strain on the health care

C. C. Herb $(\bowtie) \cdot$ J. Hertel

Department of Kinesiology, University of Virginia, 210 Emmet

St. South, Charlottesville, VA 22903, USA

e-mail: cch2jd@virginia.edu system in the form of medical and productivity expenses adds to the indirect cost of ankle sprains. Caldwell et al. [2], identified "sprains and strains" as the most common outpatient condition in the United States with 4.4 million emergency department visits per year. Nearly half of all ankle sprains occur during athletic activity (49.3\%) [3]. Following an initial ankle sprain, up to $73 \%$ of patients experience at least one subsequent ankle sprain and $59 \%$ of those patients report long term disability [4]. Swenson et al. [5] reported that, from 2005 to 2008, ankle injuries were the most common recurrent injury with $28.3 \%$ of all recurrent injuries in high school athletes. The primary risk factor for an ankle sprain remains a history of a previous sprain [6]. Between $30 \%$ and $70 \%$ of initial ankle sprains result in development of chronic ankle instability (CAI) [7]. CAI is defined as a condition resulting from a significant lateral ankle sprain with greater than 12 months of residual symptoms of "giving way" or subjective instability [8•]. The high rate of lateral ankle sprains, risk of long-term dysfunction, and high costs of health care make lateral ankle sprains a substantial concern for an active population.

Physical disability subsequent to recurrent ankle sprains has included range of motion and strength deficits, sensorimotor alterations, balance impairments, gait alterations and post-traumatic osteoarthritis [9-15]. Current research in these areas has focused on improving function and lessening disability associated with this condition, as well as identifying what leads to recurrent sprains. Current treatments include external ankle support to limit excessive motion associated with lateral ankle sprains and to increase sensory input via compression of the joint. Therapeutic exercise, including balance training, has been shown to improve balance as well as function in patients with CAI [16]. Surgical intervention has been become more 
prevalent in individuals suffering from gross mechanical instability of the joint following failed conservative treatment. All of these treatments have sought to decrease the risk of recurrent sprains and protect the joint from aberrant motion that may lead to further dysfunction.

\section{Mechanism and pathophysiology}

Lateral ankle sprains have traditionally been described as an excessive inversion and plantar flexion mechanism that results in damage to the lateral ankle ligaments [17]. Current analysis techniques, including those of real time injury mechanisms, and findings in laboratory studies, have shown the mechanism to be inversion and internal rotation of the foot $[18,19]$. These findings indicate inversion velocities of up to 1,752 degrees/second and maximum inversion angles of up to $142^{\circ}$ [20]. The injury is defined by damage to the lateral ankle ligaments. Due to the multiplanar nature of the injury, inversion and internal rotation, damage to the lateral musculature and connective tissue of the lower leg and foot may also occur [14, 21, 22]. Freeman originally assessed recurring instability of the ankle in patients following a lateral ankle sprain. This instability was identified despite no findings of clinical or radiological abnormalities in joint laxity [23]. Freeman's hypothesis was that, secondary to ankle ligament injury, proprioceptive deficits affected the muscles of the lower leg and led to deficits in function and continued "giving way" of the ankle in these patients [23].

The original description of ankle instability associated with multiple lateral ankle sprains has since been expanded and defined as CAI $[8,17,24]$. Models have been proposed to better characterize those with CAI, but due to the complexity of condition, clinical identification of these patients remains a challenge [24, 25]. More specific guidelines on the diagnosis of CAI have helped to better identify the condition. Questionnaires have been developed to specifically target the associated disability and changes in quality of life [26-29]. The foot and ankle ability measure (FAAM) and sport sub-scale (FAAM-S) look to identify deficits in function associated with foot and ankle injury and has been validated in CAI patients [27]. Scores of less than $90 \%$ on the FAAM and less than $80 \%$ on the FAAM-S may be used to characterize disability in CAI patients. The Identification of Functional Ankle Instability (IdFAI) tool is a questionnaire that has been shown to be a reliable tool, identifying those with CAI as having scores $\geq 11$ [30]. The ankle instability instrument is a subjective tool to identify feelings of ankle instability in patients that have suffered recurrent ankle sprains if five yes responses are recorded [8]. The Cumberland Ankle Instability Tool (CAIT) also looks to identify those with ankle instability.
Scores of $\leq 24$ are considered to be indicative of a patient with CAI [8]. The use of these survey tools has improved the ability of clinicians and researchers to identify and characterize patients suffering from CAI.

Laxity and mechanical instability

CAI has been associated with clinical, functional and subjective disability and includes patients with mechanical laxity of the talocrural joint. Tissue damage to the lateral ankle ligaments may lead to chronic mechanical instability identified through clinical exam. Mechanical ankle laxity is a major predictor of the development of CAI [31]. In a systematic review, Hubbard et al. [32] identified that, based on clinical stress tests to the ankle, healing of the lateral ligaments may start between six weeks and three months after initial sprain; however at up to one year follow-up, residual mechanical laxity occurred in $3-31 \%$ of patients. This timing of the healing process is often incongruent with the length of time in which patients are immobilized and return to physical activity. Specifically, clinicians often push a fast return to functional activities, knowing that the injured ligaments have not had adequate time to completely heal. Further injury prior to the completion of healing may prevent adequate scar tissue formation and ultimately lead to mechanical insufficiencies in the talocrural and subtalar joints. Alterations in arthrokinematics, joint laxity, and synovial and degenerative changes of the joints have been reported in patients with recurrent ankle sprains and CAI $[25,31,33]$. Common clinician assessment includes the use of ligamentous stress test such as the anterior drawer and talar tilt test [34]. The mechanical laxity may lead to further aberrant motion at the joint, resulting in long term osteoarthritic changes. Seventy-eight percent of cases of ankle osteoarthritis were reportedly associated with a history of recurrent lateral ankle sprains, which is a higher rate compared to other large joints [35]. Depending on the laxity and function of the joint, these individuals may require surgery to restore the joint to a more stable condition, although conservative interventions are typically attempted first. The presence of CAI is not always associated with this laxity, but long term arthrokinematics changes associated with laxity may contribute to greater joint degeneration [36].

Sensorimotor function and ankle instability

The initial damage to the lateral ankle ligaments alters the function of mechanoreceptors of these ligaments disrupting the ability to sense motion at the joint $[25,37,38]$. The muscles surrounding the joint may also be affected when damage occurs to the mechanoreceptors of the lateral ligaments affecting postural control [39, 40] and muscle 
function [41-43]. Alterations in the sensorimotor control also have been associated with kinematic and kinetic changes during gait [44-47]. Clinical deficits have been identified in dynamic and static balance [48-51] and subjective function [17]. The importance of sensory input from the structures that surround the ankle is the most commonly accepted reason for the subjective instability, as the subjective instability is present even in those individuals who do not have pronounced mechanical laxity. The multimodal input from mechanoreceptors of the ligaments, muscles, tendons, and skin all play a role in static and dynamic joint stability. The redundancy in sensing force position, joint position, stretch and tension in these sensory systems emphasize the importance of these structures in healthy individuals. Deficits in sensation of vibration [52], joint position sense $[53,54]$, and force sense $[53,55]$ have all been identified in patients with ankle instability. Healing of the lateral ligaments occurs and may restore mechanical support; however, repair of the neurological structures within this tissue is not thought to occur [25, 32].

Clinical evaluation of sensory organs would allow clinicians to better identify deficits in this population. The assessment of balance can help to identify balance deficits associated with CAI. Instrumented and non-instrumented balance assessments have been used to study stability during single-limb stance in patients with CAI [56]. Forceplate assessment of balance is considered the gold standard but is not readily used in clinical practice [56]. Deficits in balance, assessed by force-plate center of pressure measures, have consistently been reported following acute ankle sprains but not in subjects with CAI [56]. Time-toboundary measures, a more sensitive assessment, however, have identified altered postural control strategies in subjects with CAI [39]. Clinical assessment should include both dynamic and static balance. The star excursion balance test (SEBT) and balance error scoring system (BESS) has been shown to identify deficits associated with ankle instability [51, 57]. The BESS is a measurement of static balance with hands on hips during three different stances (bipedal, unipedal, and tandem) on two different surfaces (solid ground, foam pad) for six sets of $20 \mathrm{~s}$ (Fig. 1). The clinician records errors which include a loss of stable stance and hands moving off hips (Table 1) [51]. The SEBT is single limb reaching task. The patient stands with hands on hips, on the affected limb and reaches with the other leg along one of three directions, anterior, posteriormedial and posterior-lateral (Fig. 2). The reach distance is recorded and reflects the dynamic balance of the individual [58]. Assessing balance remains a useful clinical tool as it can be measured over time to show changes and has been found to be useful in assessing deficits in those with CAI when compared to healthy individuals.

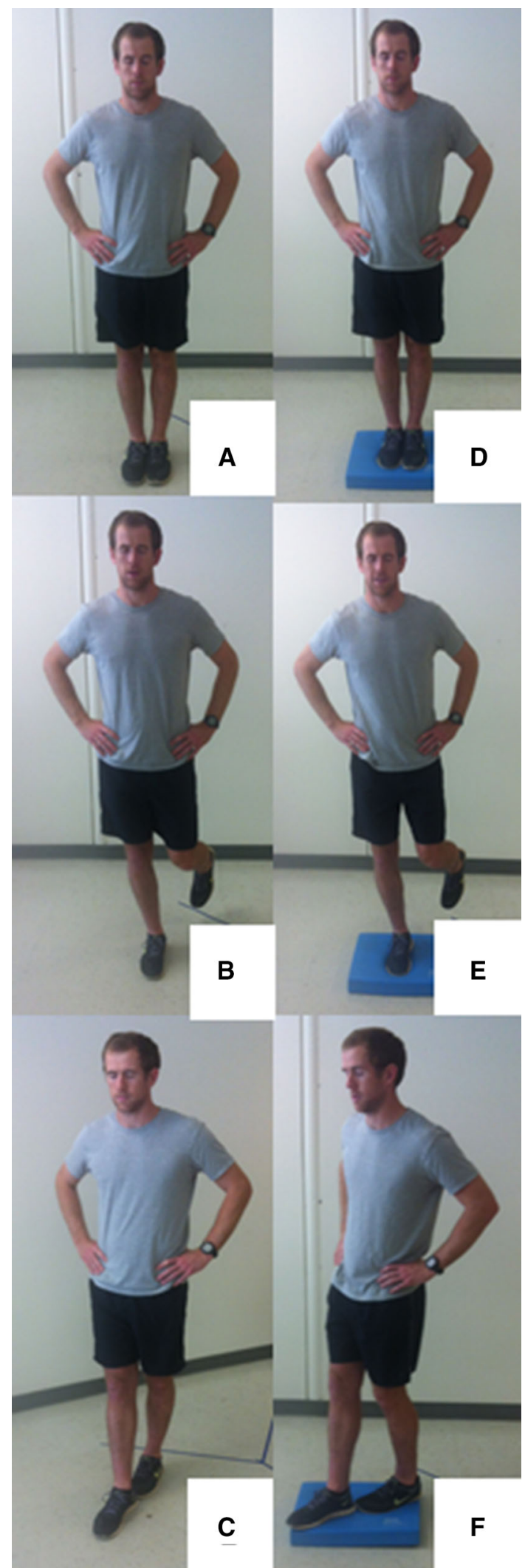

Fig. 1 Balance error scoring system has four positions in two platform conditions (Floor, foam pad). These positions include bilateral (a, d), unilateral $(\mathbf{b}, \mathbf{e})$ and tandem stance $(\mathbf{c}, \mathbf{f})$ 
Table 1 Balance error scoring system errors

\begin{tabular}{ll}
\hline Balance error scoring system errors \\
\hline 1 & Hands lifted off iliac crest \\
2 & Opening eyes \\
3 & Steps, touches down or stumbles \\
4 & Moving hip into $>30^{\circ}$ abduction \\
5 & Lifting forefoot or heel \\
6 & Remaining out of starting position $>5 \mathrm{~s}$ \\
Total errors over $20 \mathrm{~s}$ trials & \\
\hline
\end{tabular}

Errors are totaled for the six, $20 \mathrm{~s}$ trials in each stance position on each surface

Gribble et al. [59-61] has reported proximal changes in subjects with CAI during walking, jump landing and dynamic balance tasks. These changes were hypothesized to occur following changes to the ankle joint due to centrally mediated changes in neuromuscular function [25, 50]. Supraspinal changes have further been hypothesized to be the cause for differences in gait initiation [62], H-reflex modulation [63] and balance [39] in patients with CAI. These centrally mediated processes indicate that CAI patients have neuromuscular dysfunction distant from the involved ankle that may predispose them to recurrent sprains.

More recently, gait alterations have been analyzed in CAI patients. Alterations in frontal plane and sagittal plane kinematics have been identified at the ankle and knee during walking and running [33, 44-46]. Chinn et al. [46] identified a mean difference of $7.2^{\circ}$ more plantar flexion during mid-swing, and between $3.9^{\circ}$ and $5.7^{\circ}$ more inversion during stance, toe-off and swing phase between CAI and healthy controls during a jogging task. Monaghan et al. [45] reported $6^{\circ}-7^{\circ}$ more inversion prior to, during and immediately following initial contact during walking gait, while Drewes et al. [64] reported $4.83^{\circ}$ less dorsiflexion during stance phase while jogging. Associated changes in kinetics have been identified both independently and with kinematic alterations $[44,45]$. Common findings include changes in peroneal, quadriceps and gluteus medius activation as well as inverted rearfoot, plantar flexed ankle, knee sagittal motion during different periods of the gait cycle. Surface electromyography (sEMG) testing of the peroneal musculature has identified alterations in activation and timing of activation during walking gait [10, 65]. More proximal alterations in SEMG have also been identified in the gluteal musculature [66, 67] and rectus femoris [67] during walking gait. These alterations have been hypothesized to play a role in force absorption and production and associated plantar pressure research has identified differences in the center of pressure during gait [68]. Compared to healthy subjects, CAI patients have been found to have a more laterally situated center of pressure during gait $[69$, 70]. More complex analysis of movement has found more sensitive alterations in joint coupling around the ankle [47]. Drewes et al. [47] and McKeon et al. [71] identified changes in the relationship of shank transverse motion and rearfoot inversion and eversion. The analysis of joint coupling may further expand on central changes associated with coordinating gait in subjects with CAI.

Jumping and cutting tasks should be considered in those suffering from recurrent ankle sprains. Changes in ground reaction force patterns have been seen during cutting [72] and jump landing [73] with those with ankle instability having more rapid onset of vertical forces following impact. These alterations have been hypothesized to be the result of altered neuromuscular function in the lower extremity. Caulfield and Garret [73] found greater ankle dorsiflexion $\left(4.7^{\circ}-7.1^{\circ}\right)$ and knee flexion $\left(4.7^{\circ}-7.3^{\circ}\right)$ prior to, during and following landing during a single-limb landing tasks compared to healthy control subjects.
Fig. 2 Star excursion balance test. Reach directions of a anterior, b posterior lateral, c posterior medial. Average reach distance is taken over three attempts in each direction with hands on hips and stance foot flat

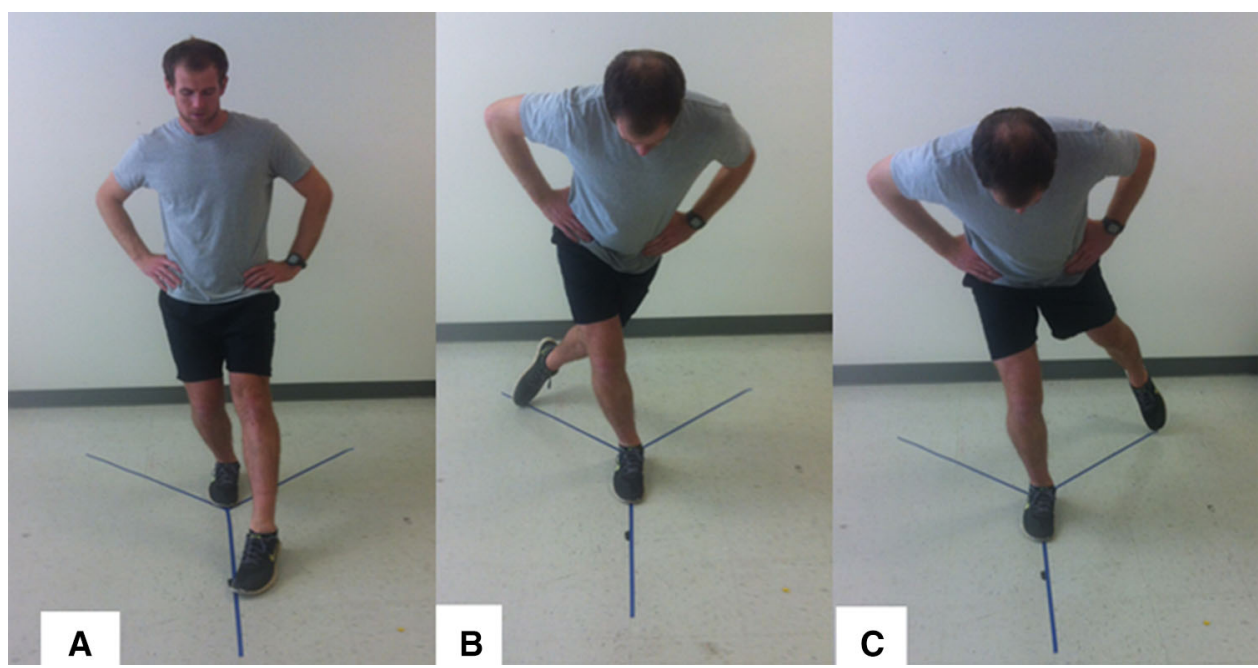


Associated differences in sEMG of the peroneus longus have also been identified during a jump landing task in patients with functional instability compared to healthy controls [74]. Alterations in sEMG of the peroneus longus, rectus femoris and gluteus medius activation were also identified during a drop jump [44]. These sEMG findings were associated with group differences in frontal plane motion at the ankle and ground reaction forces prior to and during the landing task [44].

\section{Interventions}

Acute care of recurrent sprains

Initial treatments of rest, ice, compression and elevation (RICE) have long been considered standard treatment for all acute ankle sprains. A recent systematic review of standard treatment for ankle sprains [75•], however, concluded that treatments emphasizing RICE may not be as effective as traditionally thought. Ice treatment was shown have no effect on inflammation, while, compression using elastic wraps reported mixed results on decreasing inflammation. A progressive return to activities of daily living was shown to achieve superior outcomes compared to immobilization. The use of lace-up ankle braces during return to activity had less negative outcomes including skin irritation compared to elastic wraps when used during a return to activities [75•]. Another similar guideline, PRICE, has also been used which includes protection [76]. A recent editorial [77], introduce the acronym POLICE: protection, optimal loading, ice, compression and elevation. These treatments are common in acute ankle sprains management, but there is little evidence to support or contest their use clinically.

\section{Rehabilitation}

The clinical intervention for those with a history of recurrent ankle sprains commonly consists of therapeutic exercise protocols that address identified deficits including range of motion, balance, strength, and functional training. The ultimate goal of these interventions is to decrease these limitations to reduce the risk of further lateral ankle sprains.

Changes in joint motion have been found to be associated with chronic ankle sprains. Increased anterior joint laxity [78], reduction in posterior talar glide [15], and deficits in dorsiflexion range of motion [64] have all been identified in this population. These changes in joint motion may alter the function of the joint. Basnett et al. [79] recently showed a positive correlation between dorsiflexion range of motion and dynamic balance during the SEBT. A

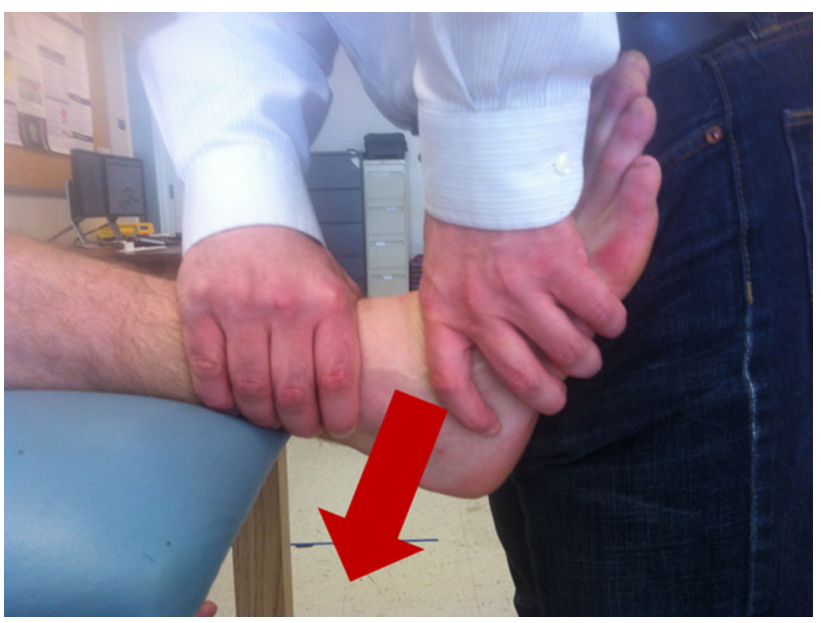

Fig. 3 Posterior talocrural joint mobilizations. Improvements in arthrokinematics and ankle range of motion have been shown with grade 3 mobilizations for 5 min three times per week

systematic review [80] recently showed improvement in dorsiflexion range of motion with static stretching but recommends clinicians be thorough in determining the cause of the dorsiflexion deficit. Anterior positional faults of the talus within the talocrural joint may result from an inversion mechanism due to the insertions of the anterior talofibular ligament [81]. These positional faults may be associated with arthrokinematics motion deficits. These limit the posterior glide of the talus in the joint during dorsiflexion [15]. Joint mobilizations have been shown to improve dorsiflexion by improving the arthrokinematics of motion [82], and these differences have also been shown to carry over acutely to landing mechanics and in postural control (Fig. 3) [83, 84]. Hoch et al. [82], showed improvements in range of motion with two weeks of grade 3 posterior talocrural mobilizations for three treatments of 5 min. Other interventions have included repositioning taping of the fibula to alter the joint structure but has not shown improvements in range of motion [85].

To address muscle weakness and function, Kaminski et al. [86] intervened with strength training and proprioceptive training on subjects with ankle instability and a history of recurrent sprains. This study used Thera-Band ${ }^{\circledR}$ elastic bands to perform inversion, eversion, dorsiflexion and plantar flexion as well as perturbation during a single limb balance exercise over a four week, 12 session rehabilitation program. There were no differences in eversion/ inversion isokinetic strength between subjects with ankle instability and healthy control subjects [86]. In contrast to this study, Docherty et al. [87, 88] found increased dorsiflexion and eversion strength following six weeks of strength training. Docherty et al. [87] found improvements in joint position sense following six weeks of strength training using elastic bands during plantar flexion, 
dorsiflexion, inversion and eversion. Three to four sets of ten repetitions were performed for $10 \mathrm{~min}$, three times per week. These changes were hypothesized to result from changes in the muscle spindles located within the musculature acting on the ankle. These alterations may have changed the gamma motor neuron function and efferent motor response [87]. Contrasting findings may be the result of different training protocols or measurement techniques; however, strength training remains a clinically useful intervention. The hypothesis that strength training may improve muscle spindle function and improve ability to sense motion and force at the joint could potentially compensate for the mechanoreceptor deficits of the fibers located within the lateral ankle ligaments. Improved muscle function including joint position sense may allow those with CAI to sense faulty ankle position and make corrections.

Balance training has long been used in rehabilitation of patients with CAI [89]. Balance training commonly involves a progression of single limb balance adding perturbations that involve removing visual input, changing surface or adding tasks such as the ball toss or reaching tasks. Dynamic balance can include more challenging tasks such as jumping. The SEBT has been shown to be a reliable test to evaluate dynamic balance but can also be used as a clinical rehabilitation exercise (Fig. 4) [90]. Balance training commonly starts in a controlled environment on a stable surface and progresses by adding different perturbations to encourage the use of multiple sensorimotor systems (Table 2). Changing the surface using foam pads or Dynadiscs ${ }^{\circledR}$, adding task constraints such as arm position or reaching tasks, or removing the input of vision add complexity to the balance task and force the individual to adapt a strategy to maintain balance [91]. These progressions should be based on the individual patient's success during the task. Observing and recording an error, for example touching down with the non-stance leg or opening eyes during an eyes closed task, can help the clinician determine a successful task. Three known studies have shown reduction in the risk of a recurrent ankle sprain following the introduction of balance exercises [92-94]. Balance training has also been shown to improve selfreported function in those with CAI following four weeks of training [16]. These findings emphasize the importance that patient reported function should play in clinical practice. Balance training improves both balance performance and patient function and should be used clinically in this population.

Gait alterations in patients suffering from recurrent sprains and CAI have been reported previously [44, 46, 95]. Gait retraining has been used in patients with several different pathologies, including total joint replacement, cerebrovascular accidents and stroke patients to develop

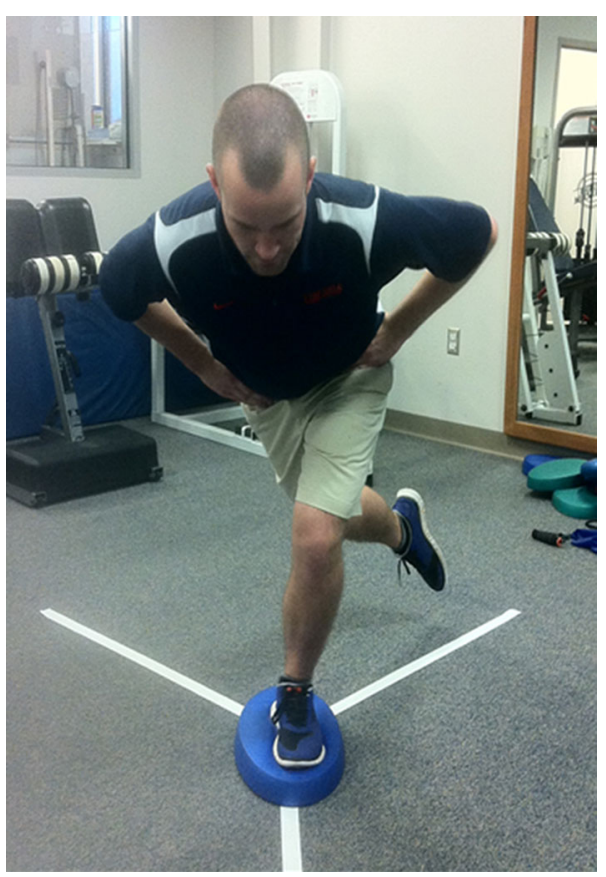

Fig. 4 An example of the SEBT being used as a dynamic balance exercise by performing reaching tasks on a foam pad

better movement strategies and avoid injurious positions or motions [96]. In patients with CAI, performing gait retraining and functional exercises that mimic daily tasks may allow individuals to develop effective and efficient movement strategies. There is limited research on interventions in this population, but clinically these exercises should replicate tasks the patient may encounter that would put them at risk for further ankle sprain including cutting, jumping and landing. Task challenges should be added to challenge the patient and help encourage strategies to overcome the task challenges.

The findings of kinematic, kinetic, sEMG, and plantar pressure during complex functional task and gait may indicate the importance of gait and functional training. Motor control strategies that include both feedback and feed-forward strategies during movement may improve patients ability to coordinate movement and avoid deleterious motions or positions. Gait training, functional jumping and cutting tasks that include the entire lower extremity may help to develop movement strategy alterations that decrease the risk of recurrent injury. Prevention programs that emphasize jump landing have been shown to decrease the risk of knee injury and similar training programs may be useful in a recurrent ankle sprain population [97].

\section{Bracing and taping}

Beyond therapeutic exercises and functional training, external stabilization devices may be able to limit motions 
Table 2 Examples of progressive static (A) and dynamic (B) balance exercises

\begin{tabular}{|c|c|c|}
\hline $\begin{array}{l}\text { Level of } \\
\text { progression }\end{array}$ & Tasks & Sets $\times$ Reps \\
\hline \multicolumn{3}{|c|}{ A. Static single limb balance progression } \\
\hline 1 & Eyes open single limb balance & $3 \times 30 s$ \\
\hline 2 & $\begin{array}{l}\text { Eyes open single limb balance on } \\
\text { foam pad }\end{array}$ & $3 \times 30 s$ \\
\hline 3 & Eyes closed single limb balance & $3 \times 30 s$ \\
\hline 4 & $\begin{array}{l}\text { Eyes closed single limb balance on } \\
\text { foam pad }\end{array}$ & $3 \times 30 s$ \\
\hline \multicolumn{3}{|c|}{ B. Dynamic balance jump to stabilization progression } \\
\hline 1 & 18 inch jump and stabilization & $1-3 \times 10$ \\
\hline 2 & $\begin{array}{l}18 \text { inch jump and stabilization with } \\
\text { hands on hips }\end{array}$ & $1-3 \times 10$ \\
\hline 3 & $\begin{array}{l}18 \text { inch jump and stabilization on } \\
\text { foam pad }\end{array}$ & $1-3 \times 10$ \\
\hline 4 & 24 inch jump and stabilization & $1-3 \times 10$ \\
\hline 5 & $\begin{array}{l}24 \text { inch jump and stabilization with } \\
\text { hands on hips }\end{array}$ & $1-3 \times 10$ \\
\hline 6 & $\begin{array}{l}24 \text { inch jump and stabilization on } \\
\text { foam pad }\end{array}$ & $1-3 \times 10$ \\
\hline 7 & 30 inch jump and stabilization & $1-3 \times 10$ \\
\hline 8 & $\begin{array}{l}30 \text { inch jump and stabilization with } \\
\text { hands on hips }\end{array}$ & $1-3 \times 10$ \\
\hline 9 & $\begin{array}{l}30 \text { inch jump and stabilization on } \\
\text { foam pad }\end{array}$ & $1-3 \times 10$ \\
\hline
\end{tabular}

Perturbation or task challenges are added as patient improves. Progression should be based on success of the task with minimal touchdowns of non-stance leg, movements of hands off hips or stance foot displacement

or alter the sensorimotor function around the joint. Ankle taping and bracing has been shown to reduce the risk of lateral ankle sprains [98]. McGuine et al. [99•, 100•], recently found that lace-up ankle braces substantially decreased the risk of lateral ankle sprains, both first-time and recurrent sprains, in a high school athlete population but did not find a difference in the severity of ankle sprains.

\section{Failed conservative treatment}

Mechanical instability, if excessive, may require intervention beyond taping and bracing and following failed conservative management. Modern surgical techniques, including the Brostrom-Gould technique, have shown positive outcomes in creating a more stable ankle joint altering the athrokinematic motion of the subtalar joint [36]. The repair consists of anatomical reconstruction of the lateral ankle ligaments to the lateral malleolus and the suturing of the peroneal retinaculum to the lateral malleolus over the lateral ligaments to reinforce the lateral capsule. The procedure may decrease the risk of developing long term changes including osteoarthritis by returning the ankle joint to a more stable condition.

\section{Future research}

The impact of treatment on function of patients with CAI commonly looks to identify the specific role of those treatments on the condition. Clinical practice, however, rarely performs therapy in isolation. Research on comprehensive rehabilitation may reveal an interaction of common clinical treatments.

The goal of current interventions has been to decrease the risk of ankle sprain or decrease the negative symptoms associated with the condition. Ultimately, such a goal returns these subjects to the level of function they had prior to the development of the condition. Despite all that has been done, the condition remains highly prevalent and costly. Continued research on gait changes may identify further motor control processes that lead to continued instability with the ultimate goal decreasing the presence of functional instability. Contemporary motor control theory describes the ability of the human central nervous system to process available information from the periphery and process coordinated movement that is efficient and effective. Better motor control would allow these individuals to protect the ankle joint from an inversion mechanism and protect the joint from injury. CAI has been associated with altered motor control and changes in the ability to coordinate routine movement patterns [45]. Further research specifically targeting this pathology and the motor control strategies that coordinate movement may reveal more regarding why changes occur following the development of $\mathrm{CAI}$ and how these changes can benefit from intervention to prevent the development of CAI or lessen the disability associated with the pathology.

\section{Conclusion}

The presence of lateral ankle sprains in an active population is very common and a history of an ankle sprain remains the number one risk for further ankle sprain. The multi-faceted nature of recurrent ankle sprains and CAI is associated with varying degrees of instability. Both mechanical and functional instability are associated with medical, financial and social costs on the individuals that suffer from the condition. Individuals suffering from recurrent ankle sprains can present with alterations in balance, postural control, neuromuscular function, subjective function and long term joint health changes. Identification of the pathology is primarily directed at identifying associated disability following a history of 
recurrent sprains. Joint stability exams, range of motion, strength, balance, and gait assessments, along with questionnaires, present clinical tools for identifying those with CAI, who may be at risk for recurrent sprains. Common interventions following the identification includes dynamic and static balance training, strength, manual therapy as well as more functional gait retraining and jump landing drills. External support like taping and bracing has been shown to decrease the risk of sprains. In individuals with joint laxity due to the damage to the lateral ankle ligaments, lateral ankle reconstruction may be necessary and has been shown to improve joint stability and return the joint to a more normal structure. These tools should be used along with a history to assess the risk of recurrent sprains.

\section{Compliance with Ethics Guidelines}

Conflict of Interest C.C. Herb declares no conflicts of interest. J. Hertel declares no conflicts of interest

Human and Animal Rights and Informed Consent This article does not contain any studies with human or animal subjects performed by any of the authors.

\section{References}

Papers of particular interest, published recently, have been highlighted as:

- Of importance

1. Hupperets MD, et al. Potential savings of a program to prevent ankle sprain recurrence: economic evaluation of a randomized controlled trial. Am J Sports Med. 2010;38(11):2194-200.

2. Caldwell N, et al. "How much will I get charged for this?" Patient charges for top ten diagnoses in the emergency department. PLoS One. 2013;8(2):e55491.

3. Waterman BR, et al. The epidemiology of ankle sprains in the United States. J Bone Joint Surg Am. 2010;92(13):2279-84.

4. Yeung MS, et al. An epidemiological survey on ankle sprain. $\mathrm{Br}$ J Sports Med. 1994;28(2):112-6.

5. Swenson DM, et al. Patterns of recurrent injuries among US high school athletes, 2005-2008. Am J Sports Med. 2009;37(8): 1586-93.

6. Milgrom C, et al. Risk factors for lateral ankle sprain: a prospective study among military recruits. Foot Ankle. 1991;12(1): 26-30.

7. Garrick JG, Requa RK. The epidemiology of foot and ankle injuries in sports. Clin Sports Med. 1988;7(1):29-36.

8. Gribble PA et al. Selection criteria for patients with chronic ankle instability in controlled research: a position statement of the international ankle consortium. J Orthop Sports Phys Ther. 2013; 43(8):585-91. This selection criteria was put forth by the most recent International Ankle Symposium Consortium to better define and describe the pathology of Chronic Ankle Instability.

9. Anandacoomarasamy A, Barnsley L. Long term outcomes of inversion ankle injuries. Br J Sports Med. 2005;39(3):e14 discussion e14.
10. Santilli V, et al. Peroneus longus muscle activation pattern during gait cycle in athletes affected by functional ankle instability: a surface electromyographic study. Am J Sports Med. 2005;33(8):1183-7.

11. Garn SN, Newton RA. Kinesthetic awareness in subjects with multiple ankle sprains. Phys Ther. 1988;68(11):1667-71.

12. Brown CN, Bowser B, Orellana A. Dynamic postural stability in females with chronic ankle instability. Med Sci Sports Exerc. 2010;42(12):2258-63.

13. Brown CN, Mynark R. Balance deficits in recreational athletes with chronic ankle instability. J Athl Train. 2007;42(3):367-73.

14. Pietrosimone BG, Gribble PA. Chronic ankle instability and corticomotor excitability of the fibularis longus muscle. J Athl Train. 2012;47(6):621-6.

15. Denegar CR, Hertel J, Fonseca J. The effect of lateral ankle sprain on dorsiflexion range of motion, posterior talar glide, and joint laxity. J Orthop Sports Phys Ther. 2002;32(4):166-73.

16. McKeon PO, et al. Balance training improves function and postural control in those with chronic ankle instability. Med Sci Sports Exerc. 2008;40(10):1810-9.

17. Hertel J. Functional instability following lateral ankle sprain. Sports Med. 2000;29(5):361-71.

18. Fong DT, et al. A systematic review on ankle injury and ankle sprain in sports. Sports Med. 2007;37(1):73-94.

19. Fong DT, et al. Kinematics analysis of ankle inversion ligamentous sprain injuries in sports: five cases from televised tennis competitions. Am J Sports Med. 2012;40(11):2627-32.

20. Mok KM, et al. Kinematics analysis of ankle inversion ligamentous sprain injuries in sports: two cases during the 2008 Beijing Olympics. Am J Sports Med. 2011;39(7):1548-52.

21. Kannus P, Renstrom P. Treatment for acute tears of the lateral ligaments of the ankle. Operation, cast, or early controlled mobilization. J Bone Joint Surg Am. 1991;73(2):305-12.

22. Garrick JG. The frequency of injury, mechanism of injury, and epidemiology of ankle sprains. Am J Sports Med. 1977;5(6):241-2.

23. Freeman MA. Instability of the foot after injuries to the lateral ligament of the ankle. J Bone Joint Surg Br. 1965;47(4):669-77.

24. Hiller CE, Kilbreath SL, Refshauge KM. Chronic ankle instability: evolution of the model. J Athl Train. 2011;46(2):133-41.

25 . Hertel J. Functional anatomy, pathomechanics, and pathophysiology of lateral ankle instability. J Athl Train. 2002;37(4): 364-75.

26. Hiller CE, et al. The Cumberland ankle instability tool: a report of validity and reliability testing. Arch Phys Med Rehabil. 2006;87(9):1235-41.

27. Carcia CR, Martin RL, Drouin JM. Validity of the foot and ankle ability measure in athletes with chronic ankle instability. J Athl Train. 2008;43(2):179-83.

28. Simon J, Donahue M, Docherty C. Development of the identification of functional ankle instability (IdFAI). Foot Ankle Int. 2012;33(9):755-63.

29. Docherty CL, et al. Development and reliability of the ankle instability instrument. J Athl Train. 2006;41(2):154-8.

30. Donahue M, Simon J, Docherty CL. Reliability and validity of a new questionnaire created to establish the presence of functional ankle instability: the IdFAI. Athl Train Sports Health Care. 2013;5(1):38-43.

31. Hubbard TJ, et al. Contributing factors to chronic ankle instability. Foot Ankle Int. 2007;28(3):343-54.

32. Hubbard TJ, Hicks-Little CA. Ankle ligament healing after an acute ankle sprain: an evidence-based approach. J Athl Train. 2008;43(5):523-9.

33. Brown $\mathrm{C}$, et al. Individuals with mechanical ankle instability exhibit different motion patterns than those with functional ankle instability and ankle sprain copers. Clin Biomech. 2008;23(6):822-31. 
34. Seligson D, Gassman J, Pope M. Ankle instability: evaluation of the lateral ligaments. Am J Sports Med. 1980;8(1):39-42.

35. Valderrabano V, et al. Etiology of ankle osteoarthritis. Clin Orthop Relat Res. 2009;467(7):1800-6.

36. Wainright WB, et al. The effect of modified Brostrom-Gould repair for lateral ankle instability on in vivo tibiotalar kinematics. Am J Sports Med. 2012;40(9):2099-104.

37. Michelson JD, Hutchins C. Mechanoreceptors in human ankle ligaments. J Bone Joint Surg Br. 1995;77(2):219-24.

38. Takebayashi $\mathrm{T}$, et al. Mechanosensitive afferent units in the lateral ligament of the ankle. J Bone Joint Surg Br. 1997; 79(3):490-3.

39. Hertel J, Olmsted-Kramer LC. Deficits in time-to-boundary measures of postural control with chronic ankle instability. Gait Posture. 2007;25(1):33-9.

40. McKeon PO, et al. Lateral ankle ligament anesthesia significantly alters single limb postural control. Gait Posture. 2010; 32(3):374-7.

41. McVey ED, et al. Arthrogenic muscle inhibition in the leg muscles of subjects exhibiting functional ankle instability. Foot Ankle Int. 2005;26(12):1055-61.

42. Konradsen L, Ravn JB. Ankle instability caused by prolonged peroneal reaction time. Acta Orthop Scand. 1990;61(5):388-90.

43. Tropp H. Pronator muscle weakness in functional instability of the ankle joint. Int J Sports Med. 1986;7(5):291-4.

44. Delahunt E, Monaghan K, Caulfield B. Changes in lower limb kinematics, kinetics, and muscle activity in subjects with functional instability of the ankle joint during a single leg drop jump. J Orthop Res. 2006;24(10):1991-2000.

45. Monaghan K, Delahunt E, Caulfield B. Ankle function during gait in patients with chronic ankle instability compared to controls. Clin Biomech. 2006;21(2):168-74.

46. Chinn L, Dicharry J, Hertel J. Ankle kinematics of individuals with chronic ankle instability while walking and jogging on a treadmill in shoes. Phys Ther Sport. 2013. doi:10.1016/j.ptsp. 2012.10.001.

47. Drewes LK, et al. Altered ankle kinematics and shank-rear-foot coupling in those with chronic ankle instability. J Sport Rehabil. 2009; 18(3):375-88.

48. Wikstrom EA, et al. Dynamic postural stability deficits in subjects with self-reported ankle instability. Med Sci Sports Exerc. 2007;39(3):397-402.

49. McGuine TA, et al. Balance as a predictor of ankle injuries in high school basketball players. Clin J Sport Med. 2000;10(4):239-44.

50. Gribble PA, et al. The effects of fatigue and chronic ankle instability on dynamic postural control. J Athl Train. 2004; 39(4):321-9.

51. Docherty CL, Valovich TC, McLeod, Shultz SJ. Postural control deficits in participants with functional ankle instability as measured by the balance error scoring system. Clin J Sport Med. 2006;16(3):203-8.

52. Hoch MC, McKeon PO, Andreatta RD. Plantar vibrotactile detection deficits in adults with chronic ankle instability. Med Sci Sports Exerc. 2012;44(4):666-72.

53. Arnold BL, Docherty CL. Low-load eversion force sense, selfreported ankle instability, and frequency of giving way. J Athl Train. 2006;41(3):233-8.

54. Konradsen L. Factors contributing to chronic ankle instability: kinesthesia and joint position sense. J Athl Train. 2002;37(4): 381-5.

55. Docherty CL, Arnold BL, Hurwitz S. Contralateral force sense deficits are related to the presence of functional ankle instability. J Orthop Res. 2006;24(7):1412-9.

56. McKeon PO, Hertel J. Systematic review of postural control and lateral ankle instability, part I: can deficits be detected with instrumented testing. J Athl Train. 2008;43(3):293-304.
57. Hertel J, et al. Simplifying the star excursion balance test: analyses of subjects with and without chronic ankle instability. J Orthop Sports Phys Ther. 2006;36(3):131-7.

58. Olmsted LC, et al. Efficacy of the star excursion balance tests in detecting reach deficits in subjects with chronic ankle instability. J Athl Train. 2002;37(4):501-6.

59. Gribble PA, Robinson RH. An examination of ankle, knee, and hip torque production in individuals with chronic ankle instability. J Strength Cond Res. 2009;23(2):395-400.

60. Gribble PA, Robinson RH. Alterations in knee kinematics and dynamic stability associated with chronic ankle instability. J Athl Train. 2009;44(4):350-5.

61. Gribble PA, Hertel J, Denegar CR. Chronic ankle instability and fatigue create proximal joint alterations during performance of the star excursion balance test. Int J Sports Med. 2007;28(3): 236-42.

62. Hass CJ, et al. Chronic ankle instability alters central organization of movement. Am J Sports Med. 2010;38(4):829-34.

63. Kim KM, Ingersoll CD, Hertel J. Altered postural modulation of Hoffmann reflex in the soleus and fibularis longus associated with chronic ankle instability. J Electromyogr Kinesiol. 2012; 22(6):997-1002.

64. Drewes LK, et al. Dorsiflexion deficit during jogging with chronic ankle instability. J Sci Med Sport. 2009;12(6):685-7.

65. Delahunt E, Monaghan K, Caulfield B. Altered neuromuscular control and ankle joint kinematics during walking in subjects with functional instability of the ankle joint. Am J Sports Med. 2006;34(12):1970-6.

66. Webster KA, Gribble PA. A comparison of electromyography of gluteus medius and maximus in subjects with and without chronic ankle instability during two functional exercises. Phys Ther Sport. 2013;14(1):17-22.

67. Feger M, Donovan L, Hart J, Hertel J. Lower extremity muscle activation in patients with and without chronic ankle instability. J Athl Train. 2013 (in press).

68. Hopkins JT, et al. Alterations in evertor/invertor muscle activation and center of pressure trajectory in participants with functional ankle instability. J Electromyogr Kinesiol. 2012; 22(2):280-5.

69. Morrison KE, et al. Plantar pressure during running in subjects with chronic ankle instability. Foot Ankle Int. 2010;31(11): 994-1000.

70. Schmidt H, Sauer L, Yong Lee S, Saliba S, Hertel J. Increased in-shoe lateral plantar pressures with chronic ankle instability. Foot Ankle Int. 2011;32(11):1075-80.

71. McKeon PO, et al. Effects of balance training on gait parameters in patients with chronic ankle instability: a randomized controlled trial. Clin Rehabil. 2009;23(7):609-21.

72. Dayakidis MK, Boudolos K. Ground reaction force data in functional ankle instability during two cutting movements. Clin Biomech. 2006;21(4):405-11.

73. Caulfield B, Garrett M. Changes in ground reaction force during jump landing in subjects with functional instability of the ankle joint. Clin Biomech. 2004;19(6):617-21.

74. Caulfield B, Crammond T, O'Sullivan A, Reynolds S, Ward T. Altered ankle-muscle activation during jump landing in participants with functional instability of the ankle joint. JSR. 2004;12(3):30.

75. - Kerkhoffs GM, et al. Diagnosis, treatment and prevention of ankle sprains: an evidence-based clinical guideline. Br J Sports Med. 2012; 46(12):854-60. This evidence-based clinical guideline summarized the results of a series of systematic reviews that analyzed the effectiveness of common acute treatments of lateral ankle sprains including cryotherapy, compress, and immobilization. The results should help direct clinical treatment based on the research evidence. 
76. Bleakley CM, et al. The PRICE study (Protection Rest Ice Compression Elevation): design of a randomised controlled trial comparing standard versus cryokinetic ice applications in the management of acute ankle sprain [ISRCTN13903946]. BMC Musculoskelet Disord. 2007;8:125.

77. Bleakley C, Glasgow P, MacAuley D. PRICE needs updating, should we call the POLICE? Br J Sports Med. 2011;46:220-1.

78. Hubbard TJ, et al. Quantitative assessment of mechanical laxity in the functionally unstable ankle. Med Sci Sports Exerc. 2004;36(5):760-6.

79. Basnett CR, et al. Ankle dorsiflexion range of motion influences dynamic balance in individuals with chronic ankle instability. Int J Sports Phys Ther. 2013;8(2):121-8.

80. Terada M, Pietrosimone BG, Gribble PA. Therapeutic interventions for increasing ankle dorsiflexion after ankle sprain: a systematic review. J Athl Train. 2013;48(5):696-709.

81. Wikstrom EA, Hubbard TJ. Talar positional fault in persons with chronic ankle instability. Arch Phys Med Rehabil. 2010;91(8):1267-71.

82. Hoch MC, et al. Two-week joint mobilization intervention improves self-reported function, range of motion, and dynamic balance in those with chronic ankle instability. J Orthop Res. 2012;30(11):1798-804.

83. Hoch MC, McKeon PO. Joint mobilization improves spatiotemporal postural control and range of motion in those with chronic ankle instability. J Orthop Res. 2011;29(3):326-32.

84. Delahunt E, et al. Joint mobilization acutely improves landing kinematics in chronic ankle instability. Med Sci Sports Exerc. 2013;45(3):514-9.

85. Wheeler TJ, et al. Fibular taping does not influence ankle dorsiflexion range of motion or balance measures in individuals with chronic ankle instability. J Sci Med Sport. 2013;16(6): 488-92.

86. Kaminski TW, et al. Effect of strength and proprioception training on eversion to inversion strength ratios in subjects with unilateral functional ankle instability. $\mathrm{Br} \mathrm{J}$ Sports Med. 2003;37(5):410-5 discussion 415.

87. Docherty CL, Moore JH, Arnold BL. Effects of strength training on strength development and joint position sense in functionally unstable ankles. J Athl Train. 1998;33(4):310-4.

88. Smith BI, et al. Ankle strength and force sense after a progressive, 6-week strength-training program in people with functional ankle instability. J Athl Train. 2012;47(3):282-8.

89. Wortmann MA, Docherty CL. Effect of balance training on postural stability in subjects with chronic ankle instability. J Sport Rehabil. 2013;22(2):143-9.
90. Gribble PA, Hertel J, Plisky P. Using the star excursion balance test to assess dynamic postural-control deficits and outcomes in lower extremity injury: a literature and systematic review. J Athl Train. 2012;47(3):339-57.

91. McKeon PO, Hertel J. Systematic review of postural control and lateral ankle instability, part II: is balance training clinically effective? J Athl Train. 2008;43(3):305-15.

92. McGuine TA, Keene JS. The effect of a balance training program on the risk of ankle sprains in high school athletes. Am $\mathbf{J}$ Sports Med. 2006;34(7):1103-11.

93. Verhagen E, et al. The effect of a balance training programme on centre of pressure excursion in one-leg stance. Clin Biomech. 2005;20(10):1094-100.

94. Bahr R, Lian O, Bahr IA. A twofold reduction in the incidence of acute ankle sprains in volleyball after the introduction of an injury prevention program: a prospective cohort study. Scand J Med Sci Sports. 1997;7(3):172-7.

95. Wikstrom EA, et al. Gait termination control strategies are altered in chronic ankle instability subjects. Med Sci Sports Exerc. 2010;42(1):197-205.

96. Tate JJ, Milner CE. Real-time kinematic, temporospatial, and kinetic biofeedback during gait retraining in patients: a systematic review. Phys Ther. 2010;90(8):1123-34.

97. Hubscher M, et al. Neuromuscular training for sports injury prevention: a systematic review. Med Sci Sports Exerc. 2010;42(3):413-21.

98. Verhagen EA, van Mechelen W, de Vente W. The effect of preventive measures on the incidence of ankle sprains. Clin J Sport Med. 2000;10(4):291-6.

99. - McGuine TA, Brooks A \& Hetzel S The effect of lace-up ankle braces on injury rates in high school basketball players. Am J Sports Med. 2011;39(9):1840-8. Both papers by McGuine (2011, 2012) were large scale intervention studies that identified a decrease in the risk of first time and recurrent ankle sprains in high school basketball and football players. These findings should encourage the continued use of lace-up ankle braces in high school athletes.

100. $\bullet$ McGuine TA et al. The effect of lace-up ankle braces on injury rates in high school football players. Am J Sports Med. 2012;40(1):49-57. Both papers by McGuine (2011, 2012) were large scale intervention studies that identified a decrease in the risk of first time and recurrent ankle sprains in high school basketball and football players. These findings should encourage the continued use of lace-up ankle braces in high school athletes. 